\title{
Successful Hematopoietic Stem Cell Transplantation for Autosomal Recessive STAT1 Complete Deficiency
}

\author{
Shuhei Karakawa ${ }^{1} \cdot$ Maiko Shimomura $^{1} \cdot$ Sonoko Sakata ${ }^{1} \cdot$ Tadashi Matsubayashi $^{2} \cdot$ Satoshi Okada $^{1}$
}

Received: 2 October 2020 / Accepted: 11 December 2020 / Published online: 4 January 2021

(C) The Author(s), under exclusive licence to Springer Science+Business Media, LLC part of Springer Nature 2021

\section{To the Editor:}

Signal transducer and activator of transcription 1 (STAT1) is a transcription factor that mediates signal transduction via several factors, including interferon (IFN) $-\alpha / \beta$ and IFN- $\gamma$. Germline mutations in STAT1 cause the following four types of primary immunodeficiency disorders (PIDs): (1) autosomal recessive (AR) STAT1 complete deficiency, (2) AR STAT1 partial deficiency, (3) autosomal dominant (AD) STAT1 partial deficiency, and (4) STAT1 gain of function [1]. AR STAT1 complete deficiency is a rare form of PID, with only nine cases in seven families reported to date [2-6]. It is characterized by a fatal course following severe viral and mycobacterial infections based on impaired IFN- $\alpha / \beta$ and IFN- $\gamma$ responses, respectively. We previously reported a case of AR STAT1 complete deficiency due to compound heterozygous intronic mutations [4]. Herein, we describe a case of successful treatment and recovery via hematopoietic stem cell transplantation (HSCT). Long-term survival has been achieved in two of four previously reported cases in which HSCT was attempted for the treatment of AR

Shuhei Karakawa

kara1224@hiroshima-u.ac.jp

Maiko Shimomura

shimomai0105@yahoo.co.jp

Sonoko Sakata

sonokosakata1116@yahoo.co.jp

Tadashi Matsubayashi

matr@ sis.seirei.or.jp

Satoshi Okada

sokada@hiroshima-u.ac.jp

1 Department of Pediatrics, Hiroshima University Graduate School of Biomedical and Health Science, 1-2-3 Kasumi, Minami-Ku,

Hiroshima-Shi, Hiroshima 734-8551, Japan

2 Department of Pediatrics, Seirei Hamamatsu General Hospital, Hamamatsu, Japan
STAT1 complete deficiency [5-8]. Therefore, ours is the third case in which long-term survival was achieved following HSCT. Given its poor prognosis, this illness requires early diagnosis and HSCT. However, in our case, the patient underwent transplantation at an age more advanced than that reported previously because the diagnosis could not be established until intronic mutations were identified [4].

The detailed clinical manifestation before HSCT is described in a previous report [4]. Briefly, our patient developed Bacille Calmette-Guerin lymphadenitis, with osteomyelitis due to mycobacteria and severe viral infections since early infancy. In addition, the patient had a history of pyrexia developing approximately once per month from the age of 5 years. During or after pyrexia, cellulitis-like skin redness and pustules sometimes occurred, mainly in the extremities. However, the pathogen had never been identified by biopsy, culture investigation, or other tests, suggesting the presence of hyperinflammatory responses against unidentified infectious pathogen [8]. The patient was diagnosed AR STAT1 complete deficiency due to compound heterozygous mutations at the age of 5 years and was referred to our hospital for transplantation [4]. A whole-body CT scan and bone scintigraphy revealed mandibular osteomyelitis and associated cellulitis but no deep-seated abscess. Blood tests showed sustained increases in C-reactive protein, procalcitonin, and $\beta$-D-glucan levels. The causative bacteria of osteomyelitis were not identified; however, the involvement of atypical mycobacteria was suspected because the patient had a history of mediastinal lymphadenitis and osteomyelitis of the tibia due to Mycobacterium malmoense. Treatment with rifampicin, ethambutol, clarithromycin, fluconazole, amikacin, sulfamethoxazole-trimethoprim, and acyclovir failed to control the infections. No other obvious organ disorders were noted. Allogeneic bone marrow transplantation was performed with the consent of the parents. Antituberculous drugs were discontinued before starting conditioning for HSCT, but no obvious exacerbation of mandibular osteomyelitis was observed in the course of HSCT. 


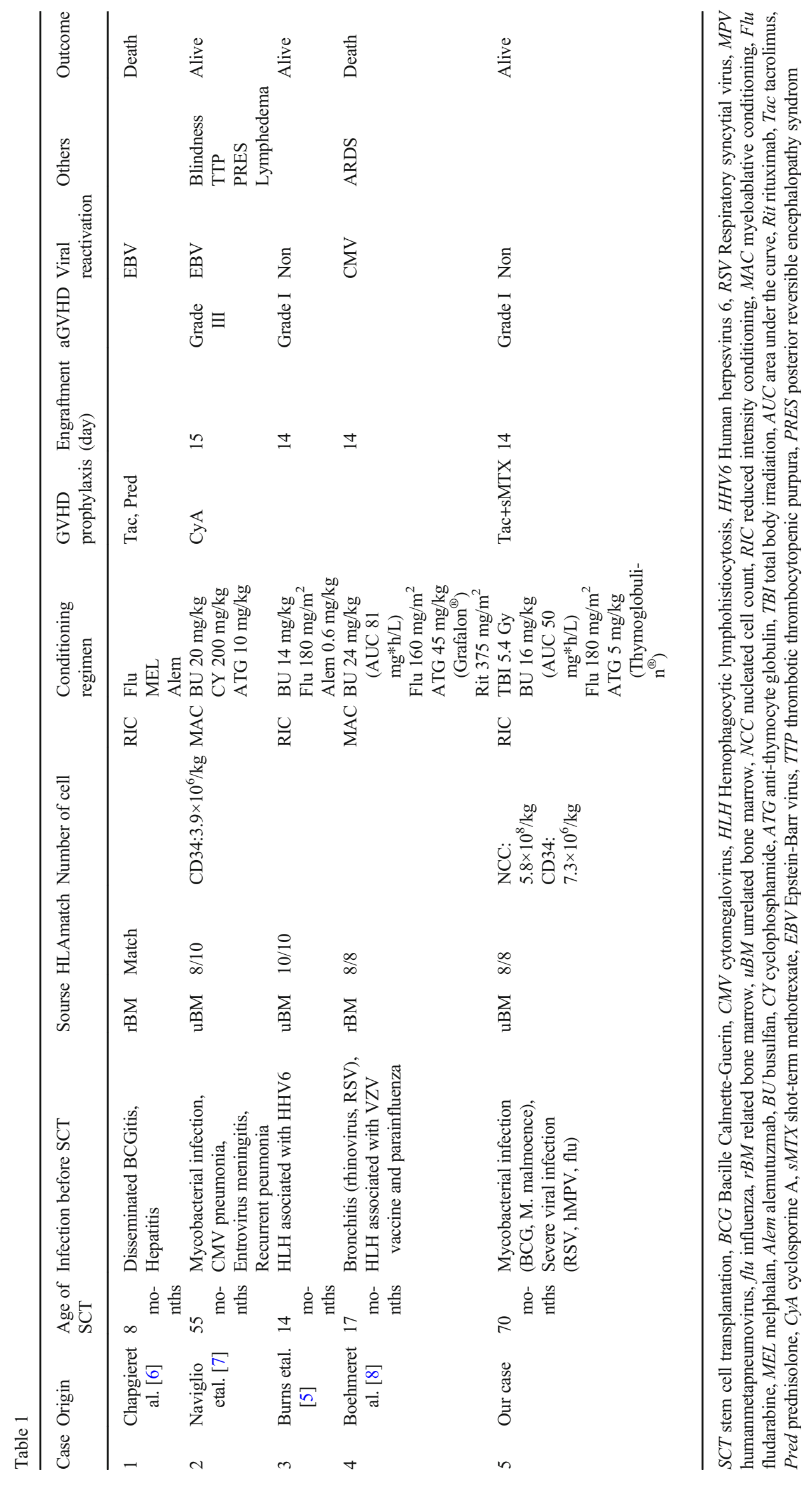


The conditioning regimen was planned as follows: total body irradiation (TBI) $3.6 \mathrm{~Gy}$, busulfan (BU) $16 \mathrm{mg} / \mathrm{kg}$ (target area under the curve, $50 \mathrm{mg} * \mathrm{~h} / \mathrm{L}$ ), fludarabine $180 \mathrm{mg} / \mathrm{m}^{2}$, and antithymocyte globulin (ATG, Thymoglobulin ${ }^{\circledR}$ ) $5 \mathrm{mg} / \mathrm{kg}$. On the day before transplantation, the numbers of white blood cells and lymphocytes were $10,000 / \mu \mathrm{L}$ and $1000 / \mu \mathrm{L}$, respectively, leading to an evaluation of insufficient myelosuppression and immunosuppression. An additional 1.8 Gy dose of TBI (5.4 Gy in total) was thus added for conditioning. The allogenic bone marrow from a human leukocyte antigen $8 / 8$ full-matched unrelated donor (CD34-positive cell count of $7.3 \times 10^{6}$ cells $/ \mathrm{kg}$ ) was used for transplantation. Tacrolimus combined with short-term methotrexate administered $15 \mathrm{mg} / \mathrm{m}^{2}$ on day 1 , and $10 \mathrm{mg} / \mathrm{m}^{2}$ on day 3,6 , and 11 was used to prevent graft-versus-host disease (GVHD).

Neutrophil and platelet engraftment were achieved on day 15 and day 86, respectively (Supplemental Fig. 1). A test for chimerism from peripheral blood revealed $88 \%$ donor type on day 14 using polymerase chain reaction of short tandem repeat. Thereafter, complete donor bone marrow chimerism was confirmed on days 30,60 , and 90 respectively. STAT1 phosphorylation analysis of peripheral blood monocytes on day 21 confirmed that the patient's peripheral blood normally responded to IFN- $\alpha$ and IFN- $\gamma$ (Supplemental Fig. 2). Various imaging procedures confirmed that mandibular osteomyelitis and associated cellulitis, which were difficult to control before transplant, were ameliorated without administration of antituberculous agents (Supplemental Fig. 3).

GVHD stage 2 of the skin was noted immediately after engraftment, but it rapidly improved after the systemic administration of prednisolone ( $1 \mathrm{mg} / \mathrm{kg} /$ day $)$ for 1 week. The persistent fever of unknown cause and recurrent eruptions continued. Thorough intensive examinations, including biopsy, were conducted with the suspicion of viral reactivation, recurrent mandibular osteomyelitis, other transplant-related infections, GVHD, and drug rash. However, no clear causes were identified. The patient spontaneously became afebrile on day 75, and skin eruptions improved on day 96. During these episodes of fever and eruption, thrombocytopenia refractory to blood transfusion was observed. No signs of thrombotic microangiopathy, other than thrombocytopenia, were found, and bone marrow examination revealed a good recovery of megakaryocytes. Splenomegaly was suspected to be one of the causes of thrombocytopenia. A spontaneous increase in thrombocytes started on day 86 . The peripheral $\mathrm{T}, \mathrm{B}$, and NK cell recoveries were confirmed on day 116. Tacrolimus was discontinued on day 240 after the transplant. There were no recurrent infections, and antiviral, antifungal, and antituberculous drugs were discontinued. At 1 year post-transplant, the patient was in a good general condition, with no transplant-related complications, such as GVHD.

AR STAT1 complete deficiency is a life-threatening form of PID. Transplantation is essential for long-term survival, but the prognosis after transplantation is not always favorable.
Among the nine AR STAT1 cases reported previously, four underwent HSCT, among whom two died with one suffering from severe post-transplantation Epstein-Barr virus reactivation with multi-organ failure and the other succumbing to cytomegalovirus reactivation with acute respiratory distress syndrome (Table 1, cases 1 and 4). Therefore, our patient joins only two others who were successfully treated with HSCT. Myeloablative conditioning was performed in two out of the four patients treated with HSCT (cases 2 and 4) [7, 8]. Longterm survival was achieved in one case who suffered from serious transplantation-related complications, including blindness (case 2) [7]. The second long-term survivor received reduced-intensity conditioning (RIC) and recovered with no complications (case 3) [5]. These two patients also received BU-based conditioning. We thus chose the BU-based RIC, but it was supplemented with low-dose TBI and ATG for prevention of rejection. We used low-dose ATG to reduce the risk of prolonged lymphopenia post-transplant that may increase the risk of viral reactivation. The engraftment was achieved relatively early. The patient was in a good general condition 1 year after HSCT, with no transplant-related complications. Though our case was successful in transplantation with BU-based conditioning, the accumulation of additional cases will be necessary to determine the appropriate conditioning regimen for AR STAT1 complete deficiency.

Our patient's delayed diagnosis was due to the difficulty in detecting intronic disease-causing in STAT1. As a result, the patient suffered from several severe and lifethreatening infections and necessarily underwent HSCT in the presence of uncontrolled mandibular osteomyelitis and associated cellulitis. The patient received transplantation at the age of 70 months, which was the oldest age among the five patients who underwent HSCT. The exacerbation of pretransplantation symptoms and the reactivation of viruses are of great concern in the case of HSCT. However, infections were well controlled throughout the entire course, and pretransplantation symptoms improved after engraftment. The current case indicates the possibility of a complete cure of this disorder by HSCT, even if the patient exhibits uncontrolled infections before transplantation.

Supplementary Information The online version contains supplementary material available at https://doi.org/10.1007/s10875-020-00948-7.

Acknowledgments We thank Michael Ciancanelli for assistance in preparing the manuscript.

Authors' Contribution Karakawa $\mathrm{S}$ wrote the manuscript. Shimomura $\mathrm{M}$ and Matsubayashi T contributed to the clinical care of the patient. Sakata $\mathrm{S}$ performed genetic and flow cytometry analysis. Okada $\mathrm{S}$ reviewed and revised the manuscript.

Funding This work was supported by Grants-in-Aid for Scientific Research from the Japan Society for the Promotion of Science (16H05355 and 19H03620), Promotion of Joint International Research from the Japan 
Society for the Promotion of Science (18KK0228), and the Practical Research Project for Rare/Intractable Diseases from Japan Agency for Medical Research and Development, AMED (JP20ek0109480, JP19ek0109209, JP16ek0109179).

\section{Compliance with Ethical Standards}

Conflict of Interest The authors declare that they have no conflicts of interest.

\section{References}

1. Okada S, Asano T, Moriya K, Boisson-Dupuis S, Kobayashi M, Casanova JL, et al. Human STAT1 gain-of-function heterozygous mutations: chronic mucocutaneous candidiasis and type I interferonopathy. J Clin Immunol. 2020;40:1065-81.

2. Dupuis S, Jouanguy E, Al-Hajjar S, Fieschi C, Al-Mohsen IZ, Al-Jumaah $\mathrm{S}$, et al. Impaired response to interferon-alpha/beta and lethal viral disease in human STAT1 deficiency. Nat Genet. 2003;33(3):388-91.

3. Vairo D, Tassone L, Tabellini G, Tamassia N, Gasperini S, Bazzoni $\mathrm{F}$, et al. Severe impairment of IFN-gamma and IFN-alpha responses in cells of a patient with a novel STAT1 splicing mutation. Blood. 2011;118(7):1806-17.

4. Sakata S, Tsumura M, Matsubayashi T, Karakawa S, Kimura S, Tamaura M, et al. Autosomal recessive complete STAT1 deficiency caused by compound heterozygous intronic mutations. Int Immunol. 2020;32:663-71.

5. Burns C, Cheung A, Stark Z, Choo S, Downie L, White S, et al. A novel presentation of homozygous loss-of-function STAT-1 mutation in an infant with hyperinflammation-a case report and review of the literature. J Allergy Clin Immunol Pract. 2016;4(4):777-9.

6. Chapgier A, Wynn RF, Jouanguy E, Filipe-Santos O, Zhang S, Feinberg J, et al. Human complete Stat-1 deficiency is associated with defective type I and II IFN responses in vitro but immunity to some low virulence viruses in vivo. J Immunol. 2006;176(8):5078-83.

7. Naviglio S, Soncini E, Vairo D, Lanfranchi A, Badolato R, Porta F. Long-term survival after hematopoietic stem cell transplantation for complete STAT1 deficiency. J Clin Immunol. 2017;37(7):701-6.

8. Boehmer DFR, Koehler LM, Magg T, Metzger P, Rohlfs M, Ahlfeld J, et al. A novel complete autosomal-recessive STAT1 LOF variant causes immunodeficiency with hemophagocytic lymphohistiocytosis-like hyperinflammation. J Allergy Clin Immunol Pract. 2020;8:3102-11.

Publisher's Note Springer Nature remains neutral with regard to jurisdictional claims in published maps and institutional affiliations. 